\title{
Haplo-deficiency of ODF1/HSPB10 in mouse sperm causes relaxation of head-to-tail linkage
}

\author{
Kefei Yang, Pawel Grzmil ${ }^{1,2}$, Andreas Meinhardt ${ }^{3}$ and Sigrid Hoyer-Fender \\ Johann-Friedrich-Blumenbach-Institute of Zoology and Anthropology - Developmental Biology, GZMB, \\ Ernst-Caspari-Haus, Justus-von-Liebig-Weg 11, Georg-August-Universität Göttingen, 37077 Göttingen, Germany, \\ ${ }^{1}$ Institute of Human Genetics, University Medicine, Heinrich-Düker-Weg 12, Georg-August-Universität Göttingen, \\ 37077 Göttingen, Germany, ${ }^{2}$ Department of Genetics and Evolution, Institute of Zoology, Jagiellonian University, \\ Gronostajowa 9, 30-387 Kraków, Poland and ${ }^{3}$ Department of Anatomy and Cell Biology, Aulweg 123, \\ Justus-Liebig-University Gießen, 35392 Gießen, Germany
}

Correspondence should be addressed to S Hoyer-Fender; Email: shoyer@gwdg.de

\begin{abstract}
The small heat shock protein ODF1/HSPB10 is essential for male fertility in mice. Targeted deletion of Odf1 resulted in acephalic sperm in homozygous mice of mixed background $(\mathrm{C} 57 \mathrm{BL} / 6 \mathrm{~J} / / 129 / \mathrm{Sv})$, whereas heterozygous animals are fully fertile. To further elucidate the function of ODF1, we generated incipient congenic mice with targeted deletion of Odf1 by successive backcrossing on the 129/Sv background. We observed that fecundity of heterozygous $\mathrm{Odf1}^{+/-}$male mice was severely reduced over backcross generations.

However, neither aberrant sperm parameters nor sperm anomalies could be observed. Ultra-structural analyses of sperm from incipient congenic heterozygous $\mathrm{Odf1}^{+/-}$males of backcross generation N7 revealed no obvious pathological findings. However, we observed an enlargement of the distance between nuclear membrane and capitulum, indicating a weakening of the sperm head-to-tail coupling. Severe male subfertility provoked by haplo-deficiency of ODF1 is therefore most probably caused by impaired head-to-tail coupling that eventually might induce sperm decapitation on the specific conditions of in vivo fertilisation. As subfertility in haplo-deficient ODF1 male mice could not be diagnosed by semen analysis, it seems to be a paradigm for unexplained infertility that is a frequent diagnosis for male fertility impairment in humans.

Reproduction (2014) 148 499-506
\end{abstract}

\section{Introduction}

Spermatozoa are highly modified germ cells specialised for the transmittance of the male genome to the oocyte. They consist of a head and a tail that are tightly interconnected in order to propel the spermatozoon head and to prevent its disconnection from the sperm tail. Firm coupling is achieved by a sophisticated structure, the connecting piece. The connecting piece is organised into nine longitudinal segmented columns that fuse cranially to form the capitulum. The capitulum is the counterpart of the basal plate, a dense structure that lines the outer nuclear membrane at the implantation fossa. At their caudal ends, the segmented columns pass into the outer dense fibres (ODFs) of the sperm tail (Fawcett 1975).

ODFs are prominent fibres found exclusively in the sperm tails of vertebrates (Fawcett 1975). They accompany the axonemal tubuli doublets on their outer site and are therefore present as a ninefold structure. ODFs most probably function in stabilising the sperm tail to promote its elastic recoil as well as to support the flagellar beat
(Baltz et al. 1990, Lindemann 1996). They are important structures as impaired development of the ODFs is a major cause of tail abnormalities in infertile men (Haidl et al. 1991). ODFs are composed of more than a dozen different proteins of which only a few have been identified (Vera et al. 1984, Oko 1988, Burfeind \& Hoyer-Fender 1991, Brohmann et al. 1997, Petersen et al. 1999). However, one major protein of the sperm tail cytoskeleton is ODF1, which localises specifically to the ODFs (Morales et al. 1994, Burmester \& Hoyer-Fender 1996).

The ODF1 protein has a molecular mass of $\sim 27 \mathrm{kDa}$ with a high content of cysteine (between 13.7 and 17\%) and proline (up to $10 \%$ ) (Van der Hoorn et al. 1990, Burfeind \& Hoyer-Fender 1991, Burfeind et al. 1993, Gastmann et al. 1993, Morales et al. 1994, Hoyer-Fender et al. 1995). Cysteine and proline residues are mainly found in repetitive $C-X-P$ tripeptide motifs present in the C-terminal end of ODF1. Albeit C-X-P repeat frequency is variable, it seems not to affect male fertility in humans (Hofferbert et al. 1993). ODF1 is conserved in evolution 
and, due to its $\alpha$-crystalline domain, ODF1 has been assigned as a small heat shock protein (sHSP) leading to renaming into HSPB10 (Kuhn et al. 1988, Gastmann et al. 1993, Petersen et al. 1999, Fontaine et al. 2003). HSPs in general are essential molecular chaperones and execute strong cytoprotective effects by preventing the aggregation of mis-folded proteins. The superfamily of sHSPs is a structural divergent group within the molecular chaperone family. Their molecular masses range from 12 to $43 \mathrm{kDa}$ and they feature the conserved $\alpha$-crystalline domain of $80-100$ amino acids. As sHSPs interact with a lot of essential cellular structures, they are implicated in a wide variety of human diseases (Sun \& MacRae 2005a,b, Hishiya \& Takayama 2008, Lanneau et al. 2008). ODF1/HSPB10 shows cell type-specific expression restricted to testis, and to be more specific to spermatids (Burmester \& Hoyer-Fender 1996). ODF1/ HSPB10 therefore seems to be functionally involved in spermatid differentiation and formation of the sperm tail.

The tight coupling between sperm head and tail is essential for successful fertilisation. Impaired formation of intimate connection between head and tail causes increased instability of head-tail junction eventually resulting in acephalic spermatozoa and human male infertility (Chemes et al. 1999, Chemes 2012). Although well-characterised ultra-structurally, the molecular components responsible for the formation of the connecting piece and the tight linkage of sperm head to tail are scarcely known. Disruption of head-tail coupling and sperm decapitation has been reported in mice mutated in Centrobin and IFT88 respectively (Liska et al. 2009, Kierszenbaum et al. 2011). We have recently demonstrated that ODF1 is essential for male fertility in mice. By deletion of the single copy Odf1 gene in mice, we could show that complete ablation of ODF1 on mixed genetic background resulted in acephalic sperm, whereas heterozygosity affected only sperm motility (Hoyer-Fender et al. 1995, Yang et al. 2012). Therefore, ODF1 has been figured out as an essential component of the sperm head-to-tail linkage apparatus. However, while ODF1-depleted mice (Odf1 ${ }^{-/}$) suffer from sperm decapitation, ultra-structural analyses of the connecting piece region were uninformative (Yang et al. 2012). Given that the genetic background has an important impact on the phenotype, we envisioned that congenic Odf1-deficient mice might be more useful for in-depth analysis of the connecting piece region. For generation of congenic Odf1-deficient strain, mice carrying the Odf1-knock out allele on mixed background (C57BL/6]//129/Sv) were backcrossed with 129/ Sv mice for up to seven generations (N7). We observed that, in heterozygous $O d f 1^{+/-}$male mice, fecundity was severely reduced over backcross generations. However, sperm parameters are similar to WT $129 / \mathrm{Sv}$ mice. Additionally, ultra-structural analyses of sperm tails and connecting piece regions isolated from incipient congenic heterozygous Odf1 ${ }^{+/-}$males (generation N7) revealed no obvious aberrations. However, there is a 1.3-fold enlargement of the distance between the nuclear membrane and the capitulum, indicating a weakening of the sperm head-to-tail linkage that most probably caused subfertility.

As a reduced amount of ODF1 did neither affect the sperm parameters nor caused obvious ultra-structural anomalies, fertility impairment would be diagnosed as unexplained. In human, unexplained infertility is a frequent diagnosis for male fertility impairment. Our results suggest that a reduction of ODF1 protein may be involved in some cases of severe male subfertility or infertility in human as well. Subtle aberrations, for e.g. the distance between nuclear membrane and capitulum, have therefore to be considered when obvious pathological findings are missing in unexplained male factor infertility.

\section{Materials and methods \\ Backcrosses and genotyping}

Generation of mice with targeted deletion of Odf1 has been described previously (Yang et al. 2012). Chimeric mice were produced by microinjection of homologous recombined ES cells into blastomeres of 3.5-day-old embryos of C57BL/6] mice and in vivo development. Heterozygous Odf1-deficient mice generated by mating of chimeric male mice with C57BL/ 6) females were backcrossed with mice from inbred strain 129/ Sv. Heterozygous offspring were backcrossed with $129 /$ Sv mice up to generation N7 that is then supposed to be incipient congenic. For genotyping, genomic DNA from tail tip biopsies was isolated using Viagen DirectPCR-Tail (Viagen Biotech, Los Angeles, CA, USA) and Proteinase $\mathrm{K}$ digestion overnight. Genotyping was performed with the primer pair Odf1-I (ATCAACTCTGCCTGAGAC)/Neomy (CCTTCTATCGCCTCCTTGACG) for detection of the neomycin cassette in the recombined allele. All mouse experiments were reviewed and approved by the local ethics commission. Licence for animal experiments was obtained by the Institute of Human Genetics.

\section{Fertility test}

The insemination capacity of the incipient congenic heterozygous Odf1-deficient males (129/Sv; generation N7) was investigated by mating with WT females. Females were checked for the presence of a vaginal plug. Liquid was isolated from uteri and examined for the presence of sperm.

\section{Sperm analysis}

Heterozygous Odf1-deficient male mice of the incipient congenic background (generation N7), as well as WT males of $129 /$ Sv strain, were used for sperm analyses. Epididymides were collected and dissected in Tyrode's medium. The sperm count in caput and cauda epididymidis was determined using the Neubauer cell counting chamber. 


\section{Sperm motility analysis}

Epididymides of three WT and three heterozygous Odf1deficient males, respectively, were dissected in IVF medium (Medi-Cult, Jyllinge, Denmark). Spermatozoa were allowed to swim out of the epididymides and were incubated for $1.5 \mathrm{~h}$ at $37^{\circ} \mathrm{C}$. Thirteen microlitres of the sperm suspension were transferred to the incubation chamber, which was set at a temperature of $37^{\circ} \mathrm{C}$. Sperm movement was quantified using the CEROS computer-assisted semen analysis system (version 10; Hamilton Thorne Research, Beverly, MA, USA). Then 1000-3000 spermatozoa from each individual were analysed using the following parameters: negative phase-contrast optics; recording, 60 frames/s; minimum contrast, 60; minimum cell size, six pixels; straightness (STR) threshold, $\geq 50 \%$; cut-off of the average path velocity (VAP) and straight-line velocity (VSL), 25 and $30 \mu \mathrm{m} / \mathrm{s}$ respectively; minimum progressive $\mathrm{VAP}$, $75 \mu \mathrm{m} / \mathrm{s}$; slow cells motile, no (this limit avoids counting sperm moved by others or Brownian motion and low-velocity non-progressive cells) and minimum static contrast, 15 pixels.

For statistical analysis, frequencies of the six sperm motility parameters VAP, VSL, curvilinear velocity (VCL), amplitude of lateral head displacement, beat cross frequency and STR were examined. As all analysed parameters were significantly different from normal distribution (Shapiro-Wilk's $W$ test) and could not be normalised by any transformation, the nonparametric alternative for the $t$-test, the Mann-Whitney $U$ test, was used. For statistical testing, sperm motility measurements of each parameter were pooled for each mouse type. Statistical analyses were performed by Statistica 9 (StatSoft; Inc., Tulsa, OK, USA).

\section{Electron microscopy}

Testes and epididymides were fixed in pieces by immersion in $1 \%$ glutaraldehyde in $0.1 \mathrm{M}$ phosphate buffer $(\mathrm{pH} 7.4)$ for $24 \mathrm{~h}$ followed by washing in $0.1 \mathrm{M}$ phosphate buffer ( $\mathrm{pH} 7.4$ ), postfixed with $2 \%$ osmium tetroxide, and embedded in epoxy (Epon) resin. After examination of semi-thin sections, specimens were sectioned ultrathin and examined by transmission electron microscopy. The distance between the nuclear membrane and the capitulum was measured using the Image J software.

\section{Results}

\section{Incipient congenic heterozygous male mice are subfertile}

Odf1 deficiency on C57BL/6J//129/Sv mixed background resulted in male infertility due to sperm decapitation. Additionally, Odf1-deficient sperm suffer from disorganised mitochondrial sheath and ODFs. Heterozygous males were fertile but showed reduced sperm motility (Yang et al. 2012). In order to generate a congenic Odf1-deficient mouse strain, heterozygous Odf1-deficient mice were backcrossed with WT mice of $129 / \mathrm{S} v$ strain for seven generations. We observed that litter size was dependent on the gender of the parent that carries the Odf1 knock out allele. While heterozygous $O d f 1^{+/-}$female mice from backcross generations zero to six (N0 to N6; N0 is the first mating with strain 129/Sv) gave birth to an average of five pups per mating (all up to 40 pups in 8 litters), i.e. a litter size that is in the range of WT $129 /$ Sv pairings (359 pups in 65 litters, that is, a mean litter size of 5.5), litter size of backcrosses using Odf $1^{+/-}$male mice was reduced to $\sim 50 \%$ (a total of 34 pups in 12 litters) (Fig. 1). Matings between heterozygous Odf $1^{+/-}$males and females of backcross generation N7 are severely impaired in fertility. In six matings, only two pups were born after more than 5 months of continuous cohabitation. Analysing the litter size during successive backcrossing revealed that fertility of female heterozygotes dropped to $\sim 3$ pups per litter in backcross generation N6 and N7, whereas male heterozygotes already have a reduced litter size in backcross generation N0. Moreover, albeit we found impaired fertility when incipient congenic heterozygous animals (backcross generation N7) were mated (Fig. 1), matings between heterozygous females $\left(O d f 1^{+{ }^{-}}\right)$and WT males $\left(O d f 1^{+/+}\right)$of backcross generation N7 have

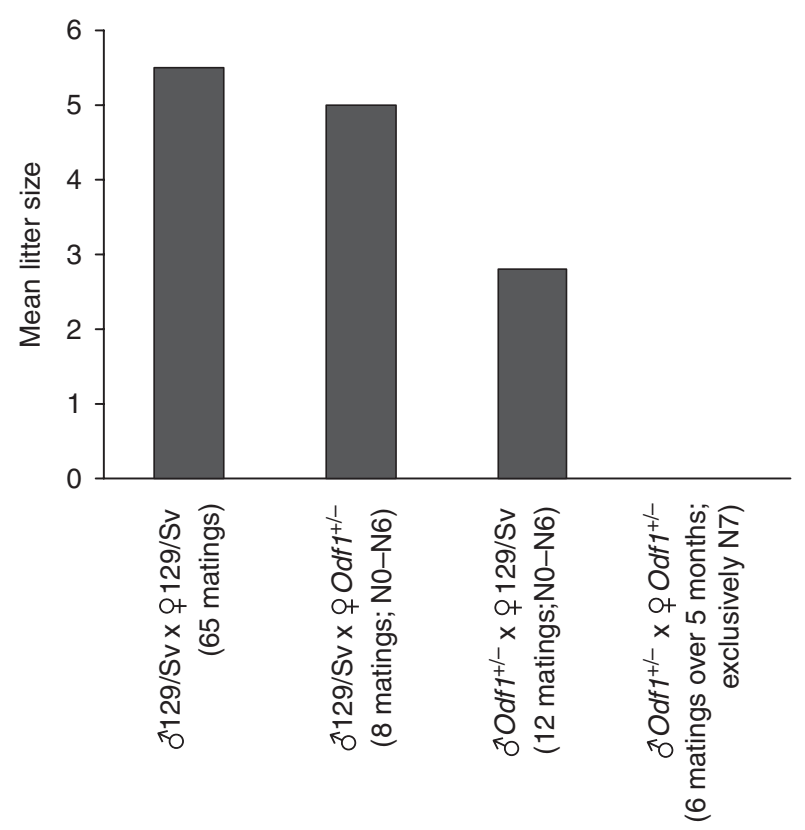

Figure 1 Impaired fertility of incipient congenic heterozygous Odf1deficient males. Litter size of matings on 129/Sv background. Matings of 129/Sv males and females gave rise to five pups per litter on an average (all in all 359 pups in 65 litters, litter size varied from 1 to 10 pups). A similar litter size was found in matings of heterozygous Odf1-deficient females with 129/Sv males from backcross generation N0 to N6 (all up to 40 pups in 8 litters, litter size varied from 2 to 8 pups). However, the litter size was reduced to $\sim 50 \%$ in backcrosses between 129/Sv females and heterozygous Odf1-deficient males (a total of 34 pups were born in 12 litters from backcross generation N0 to N6, litter size varied from 2 to 5 pups). Finally, matings between incipient congenic heterozygous Odf1-deficient mice of backcross generation N7 are severely impaired in fertility giving rise to only two pups in six matings and a continuous mating period of 5 months. 


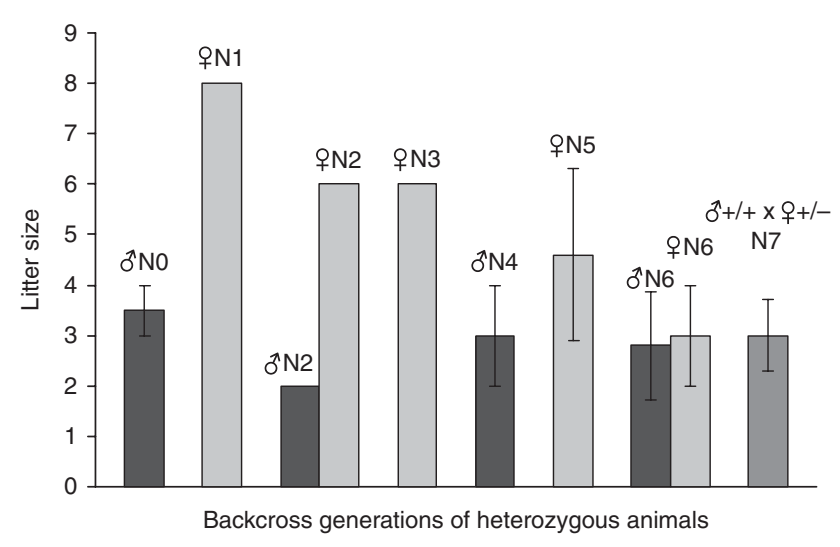

Figure 2 Impaired male fertility during successive backcrossing. Male fertility is reduced in all backcross generations, whereas female fertility steadily drops from 6 to 8 pups per litter $(\mathrm{N} 1-\mathrm{N} 3)$ to an average of 3 pups per litter in N6 and N7. Matings between incipient congenic animals (generation N7) gave birth to $\sim 3$ pups per litter when heterozygous females were mated to WT males. Heterozygous animals (either $q$ or $\delta^{*}$ ) were crossed to $129 / S_{\text {v }}$ mice and the litter size was analysed from generation N0 to N7. The litter sizes of matings with heterozygous males are represented in black, and with heterozygous females in grey. The litter size of backcross generation N7 is

represented in dark grey. S.D. in individual litter sizes are shown by bars.

a mean litter size of 3 pups (all in all 12 pups in four matings) (Fig. 2).

\section{Insemination capacity and sperm parameters are not altered in heterozygous males}

To figure out the underlying causes of reduced male fertility, mating behaviour and sperm parameters were analysed. Heterozygous male mice $(n=6$; backcross generation N7) were mated with WT 129/Sv females and females were daily examined for the presence of a vaginal plug. If insemination has occurred, females were killed, and the liquid of the uterus was obtained and examined for the presence of sperm. When sperm were present, their calculated concentrations were always in between $5 \times 10^{6}$ and $6 \times 10^{7} / \mathrm{ml}$ and no obvious sperm aberrations were found. As an exception, two mates of one specific male did not have sperm in their uteri but this situation could also be found betimes in WT mates. Copulation behaviour and insemination capability therefore seem not to be altered in heterozygous $O d f 1^{+/-}$ males. We thus asked whether sperm parameters are altered. Sperm were isolated from epididymides of heterozygous Odf $1^{+/-}$males of generation N7 as well as of WT 129/Sv males, respectively, after several weeks of sexual abstinence. We found no reduction in sperm concentration in the heterozygous condition (between 2 and $6.4 \times 10^{7} \mathrm{sperm} / \mathrm{ml} ; n=3$ ) compared with WT males (between 2.6 and $5.2 \times 10^{7} \mathrm{sperm} / \mathrm{ml} ; n=2$ ). Additionally, motility assays revealed that sperm of heterozygous males are similar to sperm of WT males even though ODF1-deficient sperm showed a higher variability especially in VAP, VSL, VCL and STR (Fig. 3).

\section{Haplo-deficiency of ODF1 increased the distance between the nuclear membrane and the capitulum}

Odf1-deficient spermatozoa on mixed background suffer from decapitation, suggesting that ODF1 is essential for the tight linkage of the spermatozoon head to the tail (Yang et al. 2012). We thus asked whether haplodeficiency of ODF1 on congenic background affects head-to-tail linkage as well, therefore accounting for the observed fertility impairment. The reduced amount of ODF1 in testicular proteins of heterozygous animals was proven by Western blot analysis (Fig. 4). Electron micrographs were taken from preparations of caput and cauda epididymidis as well as from testis of heterozygous mice (generation N7) (Fig. 5). We observed a regular arrangement of mitochondria in the mid-piece of the sperm tail (Fig. 5a and b) and a regular assembly of the ODFs (Fig. 5b). Additionally, sperm/spermatid heads are connected to their tails and no structural abnormalities of the connecting piece could be found (Fig. 5a, c and d). However, thorough analyses of the sperm-head linkage apparatus in epididymal spermatozoa (Fig. 6A) revealed that the distance between the nuclear membrane and the capitulum is increased by 1.3 -fold in congenic heterozygous males ( $n=10$ spermatozoa)
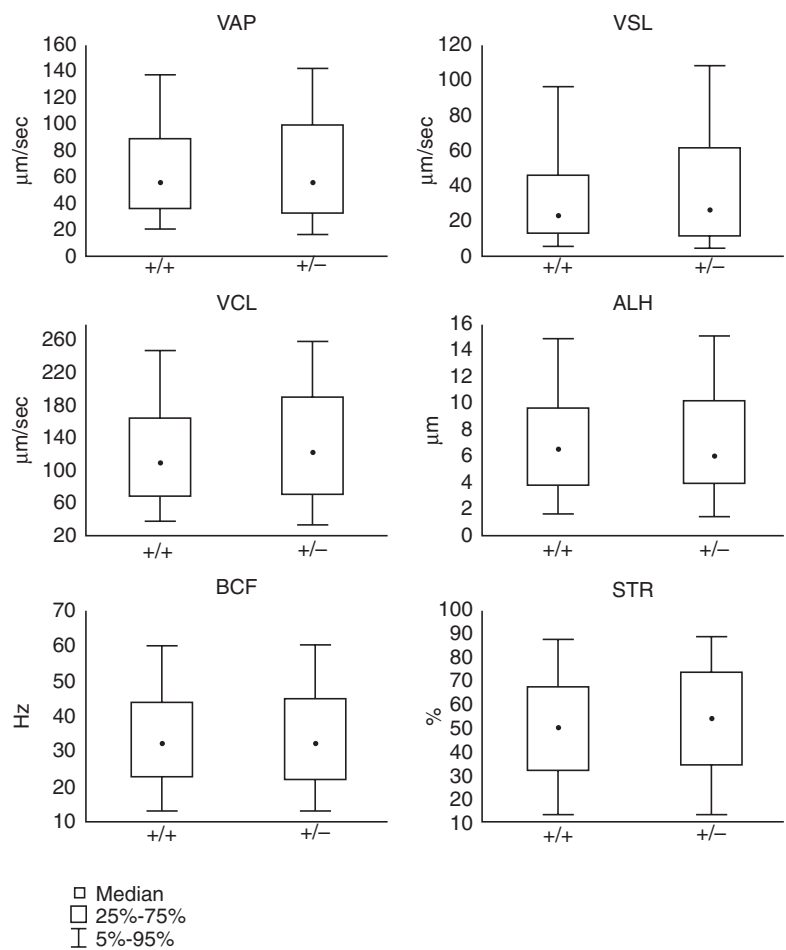

Figure 3 Computer-assisted analysis of sperm motility. The parameters of WT $(+/+)$ and heterozygous $(+/-)$ Odf1-deficient spermatozoa are shown. Average path velocity (VAP), straight-line (VSL) and curvilinear velocity (VCL) (all in $\mu \mathrm{m} / \mathrm{s})$, straightness of the movement (STR; in \%), amplitude of lateral head displacement $(\mathrm{ALH}$; in $\mu \mathrm{m})$ and beat cross frequency (BCF; in $\mathrm{Hz}$ ) were measured. For all measurements, the medians and the percentiles (25-75 and 5-95\%) are shown. 


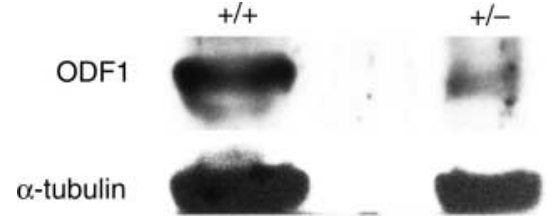

Figure 4 Reduced amount of ODF1 in $O d f 1^{+/-}$heterozygous mouse testis. Testis proteins from WT animals (+/+) and 129/Sv heterozygotes $(+/-)$ were analysed for ODF1 expression by Western blotting. Detection of $\alpha$-tubulin as the control.

compared with WT males ( $n=25$ spermatozoa) (mean values are 85 vs $68 \mathrm{~nm}$ respectively). Measurements were taken from each epididymal spermatozoon from three to six different regions of the head to tail coupling apparatus (HTCA), which span the whole HTCA and are nearly evenly distributed across it. The approximate positions of measurements are indicated in Fig. 6A. All in all 52 individual measurements were taken from heterozygous spermatozoa and 119 from WT spermatozoa. According to Student's $t$-test (two-sided and unpaired), the distances are significantly different $(P<0.005)$ (Fig. 6B).

Measurements taken from elongated spermatids of testis preparations from a heterozygous mouse revealed an even greater mean distance of $92 \mathrm{~nm} \quad(n=5$ spermatids), and those taken from a congenic WT mouse of generation N7 a mean distance of $68 \mathrm{~nm}$ ( $n=4$ spermatids), which were both not included in our calculation. To further substantiate our observation, we additionally measured the distance between the nuclear membrane and the capitulum in heterozygous $O d f 1^{+/-}$ epididymal sperm of C57BL/6J genetic background $(n=28$ spermatozoa from two individuals; all in all 94 individual measurements). Their mean distance was calculated to be of $83 \mathrm{~nm}$ that is significantly different from that of WT sperm $(P<0.005$, according to Student's $t$-test, two-sided and unpaired) (Fig. 6B).

\section{Discussion}

The sHSP ODF1/HSPB10 is essential for male fertility. We have previously reported that ODF1 depletion caused derangement of the mitochondrial sheath and of the ODFs. Moreover, ODF1 is important for the tight linkage of the sperm head to the tail causing sperm decapitation when missing (Yang et al. 2012). However, heterozygous male mice of mixed genetic background (C57BL/6)//129/Sv) are fully fertile albeit having reduced sperm motility. We show herein that incipient congenic heterozygous $O d f 1^{+/-}$male mice, generated by successive backcrossing of Odf1-depleted mice of mixed background (either heterozygous females or males) to inbred $129 / \mathrm{Sv}$ mice, are strongly reduced in fertility. In six individual matings, incipient congenic male mice of backcross generation N7 gave birth to only two pups after more than 5 months of continuous cohabitation. Moreover, the male fertility rate is already reduced to $\sim 50 \%$ in backcross matings compared with WT crosses. While fertility of heterozygous females continuously drops during progressive backcrosses, male fertility is already severely reduced in generation No. The decline in female fertility by successive backcrossing to $129 / \mathrm{Sv}$ suggests that the genetic background strongly affects fertility success. By backcrossing, alleles originating from the mixed background that are outside and unrelated to the target locus are successively lost. Those alleles might comprise modifier genes responsible for an increased litter size. Moreover, as we intended to generate congenic mice, we exclusively focused on the transmission of the target gene but not on reproductive success. Our breeding scheme thus relied on a few matings in each generation and thus might have unexpectedly selected for the mean litter size. However, severe subfertility of male mice could not be explained exclusively by the genetic background as heterozygous

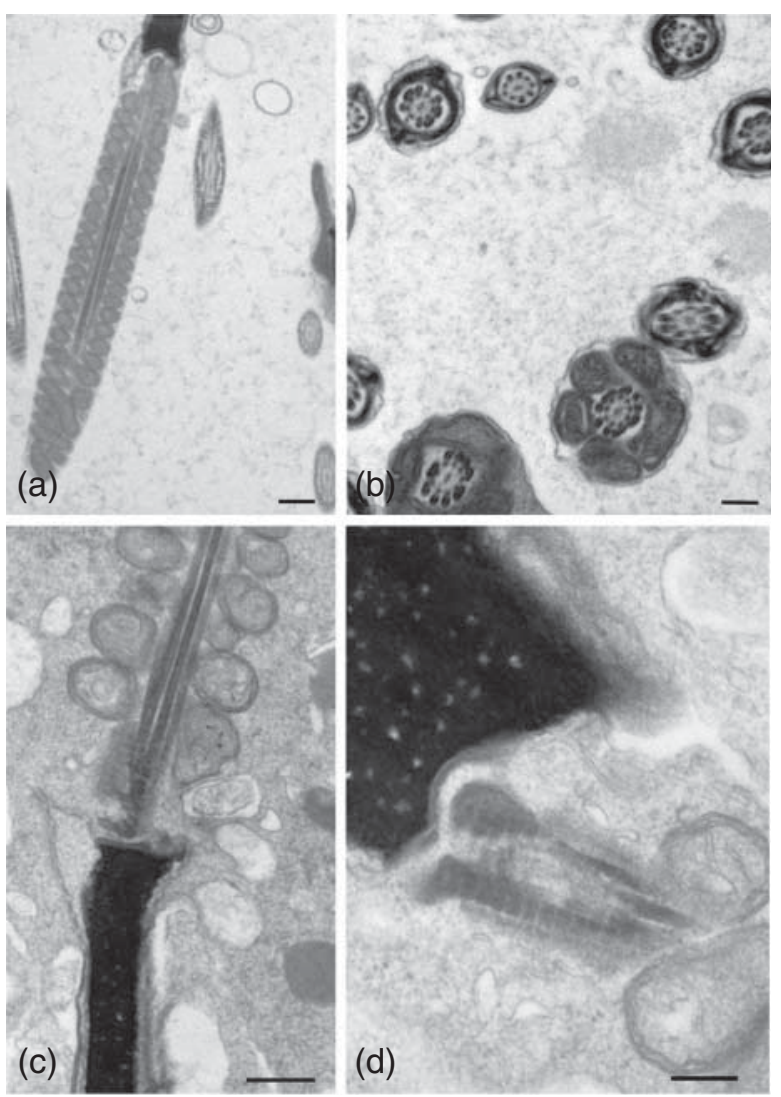

Figure 5 Ultra-structural analyses of spermatozoa/elongated spermatids from incipient congenic heterozygous $O d f 1^{+/-}$mice. Images were taken from probes isolated from caput (a) and cauda (b) epididymidis as well as from testis (c and d) demonstrating regular arrangement of the mitochondria in the mid-piece region of the sperm tail ( $a$ and $b$ ), and regular arrangement of the axonemal structure and the outer dense fibres (b). Spermatid heads are linked to their tails with a regular formation of the connecting piece (c and d). Bars are of $500 \mathrm{~nm}$ ( $a$ and c) and $250 \mathrm{~nm}$ (b and d). 

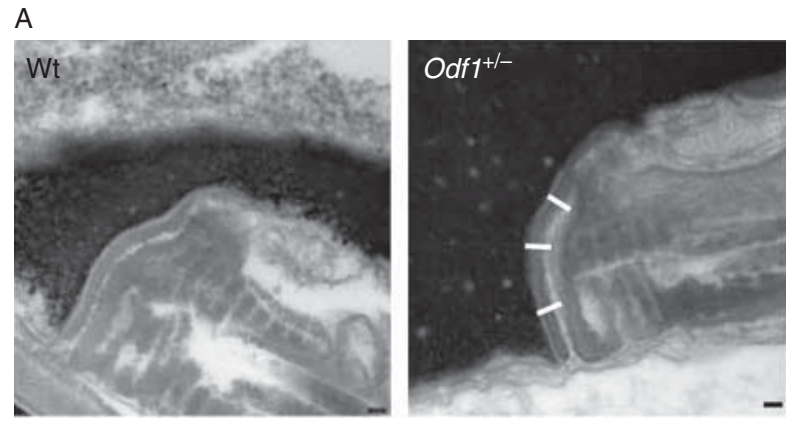

B

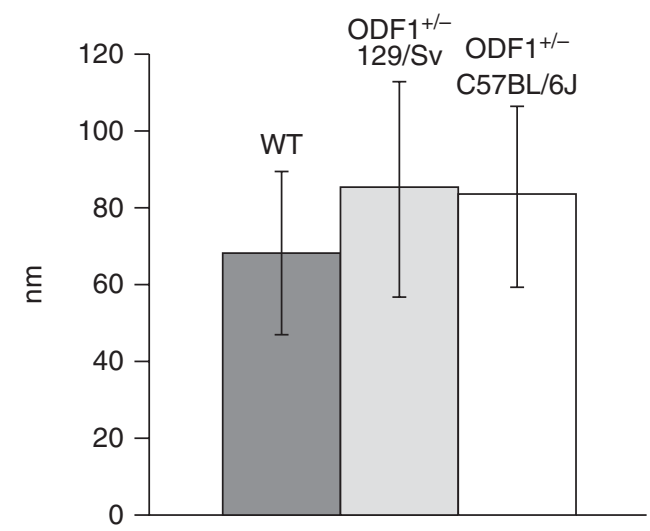

Figure 6 Relaxation of sperm head-to-tail linkage by ODF1 reduction in heterozygous $O d f 1^{+/-}$mice. (A) Electron micrographs were taken from the connecting piece region of WT and congenic heterozygous $O d f 1^{+/-}$sperm (generation N7) $\left(O d f 1^{+/-}\right)$. Apparent difference in the distance between the nuclear membrane and the capitulum was confirmed by measurement (B). In WT sperm, the mean distance is $68 \mathrm{~nm}$, whereas in congenic heterozygous Odf $1^{+/-}$males $\left(O d f 1^{+/-}\right.$ $129 / \mathrm{Sv}$ ), a mean distance of $85 \mathrm{~nm}$ was measured. In heterozygous $\mathrm{Odf1}^{+/-}$males of $\mathrm{C} 57 \mathrm{BL} / 6 \mathrm{~J}$ genetic background, a mean distance of $83 \mathrm{~nm}$ was measured (ODF1 ${ }^{+/-}$C57BL/6J). s.D.s are included. Statistical analysis by Student's $t$-test revealed significant differences $(P<0.005)$. The approximate regions used for taking measurements are marked by white bars in (A). Scale bars in electron micrographs are $50 \mathrm{~nm}$.

females of generation N7 gave birth to three pups per litter when mated to incipient congenic males of generation N7 that are WT for Odf1. Both together, severe subfertility of heterozygous male mice and reduced fertility of female mice might account for the infertility observed in generation N7 pairings. The generally much higher litter size of strain C57BL/6), on the other hand, might explain as to why haplo-deficiency of ODF1 seemed not to affect male fertility on a mixed genetic background (C57BL/6J//129/Sv) despite observed relaxation of head-tail linkage.

Although congenic heterozygous males showed more or less normal sperm parameters, for e.g. sperm count and sperm motility, and did not reveal obvious ultrastructural sperm anomalies, we noticed a significant increase in the distance between the nuclear membrane and the capitulum when ODF1 is reduced. A larger distance between the nucleus and the capitulum most probably reflects weakening of sperm head-tail linkage that eventually might provoke decapitation potentially when hyperactivated in motility in the female oviduct (Turner 2006). In men, cases of infertility have already been reported in which spermatozoa are easily decapitated when manipulated for ICSI, but whether weakening of the HTCA is causative is not known (Kamal et al. 1999). The relaxed sperm head-to-tail coupling by ODF1 reduction might also be the cause of the observed increased variability in sperm velocity. However, as sperm parameters are normal, apart from slight variations, fertility impairment would be diagnosed as 'unexplained' using routine semen analysis. In humans, unexplained infertility is a frequent diagnosis for male fertility impairment.

A congenital decapitated sperm defect, similar to that observed in ODF1-deficient sperm, was first reported in infertile bulls (Hancock \& Rollinson 1949, Blom \& Birch-Andersen 1970). In humans, the sperm decapitation defect has been casually reported but is very rare. Even though not yet confirmed, a genetic origin has been supposed (Chemes et al. 1999, Chemes \& Rawe 2010, Chemes 2012). Acephalic spermatozoa, although phenotypically similar, seem to have diverse origins as revealed by ultra-structural analyses. Head-tail detachment is provoked by specific morphological defects affecting the tight insertion of the tail into the implantation fossa. Thus, decapitation occurred at the level of the proximal centriole, resulted from the dissociation between proximal and distal centrioles, originated from defects in the implantation fossa and the basal plate or might be due to dysfunctional sperm centrioles affecting migration and positioning of the tail (Perotti et al. 1981, Baccetti et al. 1984, Holstein et al. 1986, Chemes et al. 1987, Toyama et al. 2000). ODF1 is restricted to spermatids and localised to the ODFs, capitulum and basal plate, thus implying that its function is required only in the germ cell and specifically in headtail coupling (Burmester \& Hoyer-Fender 1996, Schalles et al. 1998, Yang et al. 2012). It is currently not known whether loss of ODF1 affects sperm centriolar functions or the tight attachment of the capitulum to the implantation fossa. But the regular morphology of the connecting piece in haplo-deficient ODF1 sperm together with the observed enlargement of the distance between the nuclear membrane and the capitulum suggested that ODF1 mediated the linkage between the capitulum and the nucleus without affecting centriolar function.

Although there are currently several mouse mutants with the decapitated sperm defect, it is arguable whether they are related to the human defects as no genetic alterations in humans with acephalic sperm have been demonstrated. Morphological and motility defects with a frequent loss of the sperm head have been described in sperm deficient for sperm-associated antigen 6 (SPAG6), 
the homologue of the Chlamydomonas PF16 locus encoding a protein of the central apparatus of the axoneme (Neilson et al. 1999, Sapiro et al. 2000, 2002). Easy sperm decapitation was also reported in mice with Oaz-t (Oaz3) deficiency, the testis-specific ornithine decarboxylase antizyme, presumably by affecting the local concentration of polyamines (Tokuhiro et al. 2009). Finally, expression of a truncated isoform of Centrobin (Cntrob) by insertion of an endogenous retrovirus into intron 10 of the rat Cntrob gene was identified in the rat $h d$ mutation causing male infertility. Expression of the truncated Centrobin in spermatids provoked disruption of the head-tail coupling apparatus resulting in sperm decapitation and male infertility (Liska et al. 2009). Centrobin (that is also named NIP2) is ubiquitously expressed and associated specifically with the daughter centriole that gives rise to the proximal centriole in spermatids. Centrobin depletion by small interfering RNA inhibited centriole duplication in cell culture (Zou et al. 2005). Centrobin/ NIP2 interacts with tubulin and stabilises the microtubule structure. Thus, by stabilising the nascent centriole structure, Centrobin allows for centriole duplication (Jeong et al. 2007, Lee et al. 2010, Gudi et al. 2011). The impact of Centrobin in sperm head-tail coupling, therefore, is most probably provoked by its microtubule stabilising function. By contrast, the specific association of ODF1 with the HTCA turns it into an outstanding candidate for a putative linker function in the HTCA.

How might ODF1 mediate head-tail linkage? ODF1 shows weak self-association and binds to several other proteins (Shao \& van der Hoorn 1996). Interaction with ODF2, the main scaffolding protein of the ODFs, which also localised to the connecting piece, might enable tight binding of the sperm tail via the connecting piece (Shao et al. 1997, Petersen et al. 1999). However, binding partners that mediate the connection to the nucleus at the implantation fossa via the basal plate are currently not known. Among the identified ODF1-interacting proteins, none is located at a position to possibly function in sperm head-tail coupling (Shao et al. 1999, Bhullar et al. 2003, Zarsky et al. 2003, Zhang et al. 2004, Fitzgerald et al. 2005). The basal plate and its intrinsic proteins are crucial when looking for the head-tail coupling apparatus. However, it remains a challenge for the future to unravel the molecular composition of the sperm head-tail coupling apparatus and to figure out protein-protein interactions. This would be an important prerequisite for the targeted search of the genetic origins of male factor infertility caused by sperm decapitation.

\section{Declaration of interest}

The authors declare that there is no conflict of interest that could be perceived as prejudicing the impartiality of the research reported.

\section{Funding}

This work was supported by the Deutsche Forschungsgemeinschaft (HO 1440/13-1 and 13-2) to S Hoyer-Fender.

\section{Acknowledgements}

The authors gratefully acknowledge animal husbandry by Stefan Wolff and Lea Piontek and the expert technical assistance of Gerd Kripp.

\section{References}

Baccetti B, Selmi MG \& Soldani P 1984 Morphogenesis of decapitated spermatozoa in a man. Journal of Reproduction and Fertility 70 395-397. (doi:10.1530/jrf.0.0700395)

Baltz JM, Williams PO \& Cone RA 1990 Dense fibers protect mammalian sperm against damage. Biology of Reproduction 43 485-491. (doi:10. 1095/biolreprod43.3.485)

Bhullar B, Zhang Y, Junco A, Oko R \& van der Hoorn FA 2003 Association of kinesin light chain with outer dense fibers in a microtubule-independent fashion. Journal of Biological Chemistry 278 16159-16168. (doi:10. 1074/jbc.M213126200)

Blom E \& Birch-Andersen A 1970 Ultrastructure of the decapitated sperm defect in Guernsey bulls. Journal of Reproduction and Fertility 23 67-72. (doi:10.1530/jrf.0.0230067)

Brohmann H, Pinnecke S \& Hoyer-Fender S 1997 Identification and characterization of new cDNAs encoding outer dense fiber proteins of rat sperm. Journal of Biological Chemistry 272 10327-10332. (doi:10.1074/ jbc.272.15.10327)

Burfeind P \& Hoyer-Fender S 1991 Sequence and developmental expression of a mRNA encoding a putative protein of rat sperm outer dense fibers. Developmental Biology 148 195-204. (doi:10.1016/00121606(91)90329-2)

Burfeind P, Belgardt B, Szpirer C \& Hoyer-Fender S 1993 Structure and chromosomal assignment of a gene encoding the major protein of rat sperm outer dense fibers. European Journal of Biochemistry 216 497-505. (doi:10.1111/j.1432-1033.1993.tb18168.x)

Burmester S \& Hoyer-Fender S 1996 Transcription and translation of the outer dense fiber gene (Odf1) during spermiogenesis in the rat. A study by in situ analyses and polysome fractionation. Molecular Reproduction and Development 45 10-20. (doi:10.1002/(SICl)1098-2795(199609) 45:1 < 10::AID-MRD2 > 3.0.CO;2-V)

Chemes HE 2012 Sperm centrioles and their dual role in flagellogenesis and cell cycle of the zygote. In The Centrosome, pp 33-48. Ed. H Schatten. Springer: Humana Press.

Chemes HE \& Rawe VY 2010 The making of abnormal spermatozoa: cellular and molecular mechanisms underlying pathological spermiogenesis. Cell Tissue Research 341 349-357. (doi:10.1007/s00441-010-1007-3)

Chemes HE, Carizza C, Scarinci F, Brugo S, Neuspiller N \& Schwarsztein L 1987 Lack of a head in human spermatozoa from sterile patients: a syndrome associated with impaired fertilization. Fertility and Sterility 47 310-316.

Chemes HE, Puigdomenech ET, Carizza C, Olmedo SB, Zanchetti F \& Hermes R 1999 Acephalic spermatozoa and abnormal development of the head-neck attachment: a human syndrome of genetic origin. Human Reproduction 14 1811-1818. (doi:10.1093/humrep/14.7.1811)

Fawcett DW 1975 The mammalian spermatozoon. Developmental Biology 44 394-436. (doi:10.1016/0012-1606(75)90411-X)

Fitzgerald CJ, Oko RJ \& van der Hoorn FA 2005 Rat Spag5 associates in somatic cells with endoplasmic reticulum and microtubules but in spermatozoa with outer dense fibers. Molecular Reproduction and Development 73 92-100. (doi:10.1002/mrd.20388)

Fontaine JM, Rest JS, Welsh MJ \& Benndorf R 2003 The sperm outer dense fiber protein is the 10 th member of the superfamily of mammalian small stress proteins. Cell Stress and Chaperones 8 62-69. (doi:10.1379/14661268(2003)8<62:TSODFP $>2.0$. CO;2)

Gastmann O, Burfeind P, Günther E, Hameister H, Szpirer C \& HoyerFender S 1993 Sequence, expression, and chromosomal assignment of a human sperm outer dense fiber gene. Molecular Reproduction and Development 36 407-418. (doi:10.1002/mrd.1080360402) 
Gudi R, Zou C, Li J \& Gao Q 2011 Centrobin-tubulin interaction is required for centriole elongation and stability. Journal of Cell Biology 193 711-725. (doi:10.1083/jcb.201006135)

Haidl G, Becker A \& Henkel R 1991 Poor development of outer dense fibers as a major cause of tail abnormalities in the spermatozoa of asthenoteratozoospermic men. Human Reproduction 6 1431-1438.

Hancock JL \& Rollinson DHL 1949 A seminal defect associated with sterility of Guernsey bulls. Veterinary Record 61 742-743.

Hishiya A \& Takayama S 2008 Molecular chaperones as regulators of cell death. Oncogene 27 6489-6506. (doi:10.1038/onc.2008.314)

Hofferbert S, Burfeind P, Hoyer-Fender S, Lange R, Haidl G \& Engel W 1993 A homozygous deletion of 27 basepairs in the coding region of the human outer dense fiber protein gene does not result in a pathologic phenotype. Human Molecular Genetics 2 2167-2170. (doi:10.1093/ hmg/2.12.2167)

Holstein AF, Schill WB \& Breucker H 1986 Dissociated centriole development as a cause of spermatid malformation in man. Journal of Reproduction and Fertility 78 719-725. (doi:10.1530/jrf.0.0780719)

Hoyer-Fender S, Burfeind P \& Hameister H 1995 Sequence of mouse Odf1 CDNA and its chromosomal localization: extension of the linkage group between human chromosome 8 and mouse chromosome 15. Cytogenetics and Cell Genetics 70 200-204. (doi:10.1159/000134033)

Jeong Y, Lee J, Kim K, Yoo JC \& Rhee K 2007 Characterization of NIP2/centrobin, a novel substrate of Nek2, and its potential role in microtubule stabilization. Journal of Cell Science 120 2106-2116. (doi:10.1242/jcs.03458)

Kamal A, Mansour R, Fahmy I, Serour G, Rhodes C \& Aboulghar M 1999 Easily decapitated spermatozoa defect: a possible cause of unexplained infertility. Human Reproduction 14 2791-2795. (doi:10.1093/humrep/ 14.11.2791)

Kierszenbaum AL, Rivkin E, Tres LL, Yoder BK, Haycraft CJ, Bornens M \& Rios RM 2011 GMAP210 and IFT88 are present in the spermatid golgi apparatus and participate in the development of the acrosomeacroplaxome complex, head-tail coupling apparatus and tail. Developmental Dynamics 240 723-736. (doi:10.1002/dvdy.22563)

Kuhn R, Schäfer U \& Schäfer M 1988 Cis-acting regions sufficient for spermatocyte-specific transcriptional and spermatid-specific translational control of the Drosophila melanogaster gene mst(3)gl-9. EMBO Journal 7 447-454.

Lanneau D, Brunet M, Frisan E, Solary E, Fontenay M \& Garrido C 2008 Heat shock proteins: essential proteins for apoptosis regulation. Journal of Cellular and Molecular Medicine 12 743-761. (doi:10.1111/ j.1582-4934.2008.00273.x)

Lee J, Jeong Y, Jeong S \& Rhee K 2010 Centrobin/NIP2 is a microtubule stabilizer whose activity is enhanced by PLK1 phosphorylation during mitosis. Journal of Biological Chemistry 285 25476-25484. (doi:10. 1074/jbc.M109.099127)

Lindemann CB 1996 Functional significance of the outer dense fibers of mammalian sperm examined by computer simulation with the geometric clutch model. Cell Motility and the Cytoskeleton 34 258-270. (doi:10. 1002/(SICl)1097-0169(1996)34:4<258::AID-CM1 > 3.0.CO;2-4)

Liska F, Gosele C, Rivkin E, Tres L, Cardoso MC, Domaing P, Krejci E, Snajdr P, Lee-Kirsch MA, de Rooij DG et al. 2009 Rat hd mutation reveals an essential role of centrobin in spermatid head shaping and assembly of the head-tail coupling apparatus. Biology of Reproduction 81 1196-1205. (doi:10.1095/biolreprod.109.078980)

Morales CR, Oko R \& Clermont Y 1994 Molecular cloning and developmental expression of an mRNA encoding the $27 \mathrm{kDa}$ outer dense fiber protein of rat spermatozoa. Molecular Reproduction and Development 37 229-240. (doi:10.1002/mrd.1080370215)

Neilson LI, Schneider PA, Van Deerlin PG, Kiriakidou M, Driscoll DA, Pellegrini MC, Millinder S, Yamamoto KK, French CK \& Strauss JF III 1999 cDNA cloning and characterization of a human sperm antigen (SPAG6) with homology to the product of the Chlamydomonas PF16 locus. Genomics 60 272-280. (doi:10.1006/geno.1999.5914)

Oko R 1988 Comparative analysis of proteins from the fibrous sheath and outer dense fibers of rat spermatozoa. Biology of Reproduction 39 69-182. (doi:10.1095/biolreprod39.1.169)

Perotti M-E, Giarola A \& Gioria M 1981 Ultrastructural study of the decapitated sperm defect in an infertile man. Journal of Reproduction and Fertility 63 543-549. (doi:10.1530/jrf.0.0630543)
Petersen C, Füzesi L \& Hoyer-Fender S 1999 Outer dense fiber proteins from human sperm tail: molecular cloning and expression analyses of two cDNA transcripts encoding proteins of $\sim 70 \mathrm{kDa}$. Molecular Human Reproduction 5 627-635. (doi:10.1093/molehr/5.7.627)

Sapiro R, Tarantino LM, Velazquez F, Kiriakidou M, Hecht NB, Bucan \& Strauss JF III 2000 Sperm antigen 6 is the murine homologue of the Chlamydomonas reinhardtii central apparatus protein encoded by the PF16 locus. Biology of Reproduction 62 511-518. (doi:10.1095/ biolreprod62.3.511)

Sapiro R, Kostetskii I, Olds-Clarke P, Gerton GL, Radice GL \& Strauss JF III 2002 Male infertility, impaired sperm motility, and hydrocephalus in mice deficient in sperm-associated antigen 6. Molecular and Cellular Biology 22 6298-6305. (doi:10.1128/MCB.22.17.6298-6305.2002)

Schalles U, Shao X, van der Hoorn FA \& Oko R 1998 Developmental expression of the $84-\mathrm{kDa}$ ODF sperm protein: localization to both the cortex and medulla of outer dense fibers and to the connecting piece. Developmental Biology 199 250-260. (doi:10.1006/dbio.1998.8931)

Shao X \& van der Hoorn FA 1996 Self-interaction of the major 27-kilodalton outer dense fiber protein is in part mediated by a leucine zipper domain in the rat. Biology of Reproduction 55 1343-1350. (doi:10.1095/ biolreprod55.6.1343)

Shao X, Tarnasky HA, Schalles U, Oko R \& van der Hoorn FA 1997 Interactional cloning of the $84-\mathrm{kDa}$ major outer dense fiber protein Odf84. Journal of Biological Chemistry 272 6105-6113. (doi:10.1074/ jbc.272.10.6105)

Shao X, Tarnasky HA, Lee JP, Oko R \& van der Hoorn FA 1999 Spag4, a novel sperm protein, binds outer dense-fiber protein Odf1 and localizes to microtubules of manchette and axoneme. Developmental Biology 211 109-123. (doi:10.1006/dbio.1999.9297)

Sun Y \& MacRae TH 2005a The small heat shock proteins and their role in human disease. FEBS Journal 272 2613-2627. (doi:10.1111/j.17424658.2005.04708.x)

Sun Y \& MacRae TH $2005 b$ Small heat shock proteins: molecular structure and chaperone function. Cellular and Molecular Life Sciences 62 2460-2476. (doi:10.1007/s00018-005-5190-4)

Tokuhiro K, Isotani A, Yokota S, Yano Y, Oshio S, Hirose M, Wada M, Fujita K, Ogawa Y, Okabe M et al. 2009 OAZ-t/OAZ3 is essential for rigid connection of sperm tails to heads in mouse. PLoS Genetics 5 e1000712. (doi:10.1371/journal.pgen.1000712)

Toyama Y, Iwamoto T, Yajima M, Baba K \& Yuasa S 2000 Decapitated and decaudated spermatozoa in man, and pathogenesis based on the ultrastructure. International Journal of Andrology 23 109-115. (doi:10. 1046/j.1365-2605.2000.t01-1-00217.x)

Turner RM 2006 Moving to the beat: a review of mammalian sperm motility regulation. Reproduction, Fertility, and Development 18 25-38. (doi:10. 1071/RD05120)

Van der Hoorn FA, Tarnasky HA \& Nordeen SK 1990 A new rat gene RT7 is specifically expressed during spermatogenesis. Developmental Biology 142 147-154. (doi:10.1016/0012-1606(90)90158-F)

Vera JE, Brito M, Zuvic T \& Burzio LO 1984 Polypeptide composition of rat sperm outer dense fibers. A simple procedure to isolate the fibrillar complex. Journal of Biological Chemistry 259 5970-5977.

Yang K, Meinhardt A, Zhang B, Grzmil P, Adham IM \& Hoyer-Fender S 2012 The small heat shock protein ODF1/HSPB10 is essential for tight linkage of sperm head to tail and male fertility in mice. Molecular and Cellular Biology 32 216-225. (doi:10.1128/MCB.06158-11)

Zarsky HA, Cheng M \& van der Hoorn FA 2003 Novel RING finger protein OIP1 binds to conserved amino acid repeats in sperm tail protein ODF1. Biology of Reproduction 68 543-552. (doi:10.1095/biolreprod.102.009076)

Zhang Y, Oko R \& van der Hoorn FA 2004 Rat kinesin light chain 3 associates with spermatid mitochondria. Developmental Biology 275 23-33. (doi:10.1016/j.ydbio.2004.07.014)

Zou C, Li J, Bai Y, Gunning WT, Wazer DE, Band V \& Gao Q 2005 Centrobin: a novel daughter centriole-associated protein that is required for centriole duplication. Journal of Cell Biology 171 437-445. (doi:10. 1083/jcb.200506185)

Received 24 July 2014

Accepted 7 August 2014 